\title{
Evidence of a discontinuous disk structure around the Herbig Ae star HD 139614^ (Corrigendum)
}

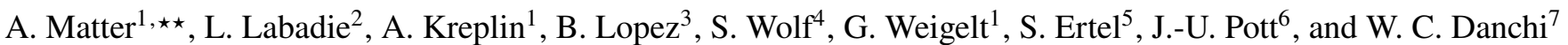

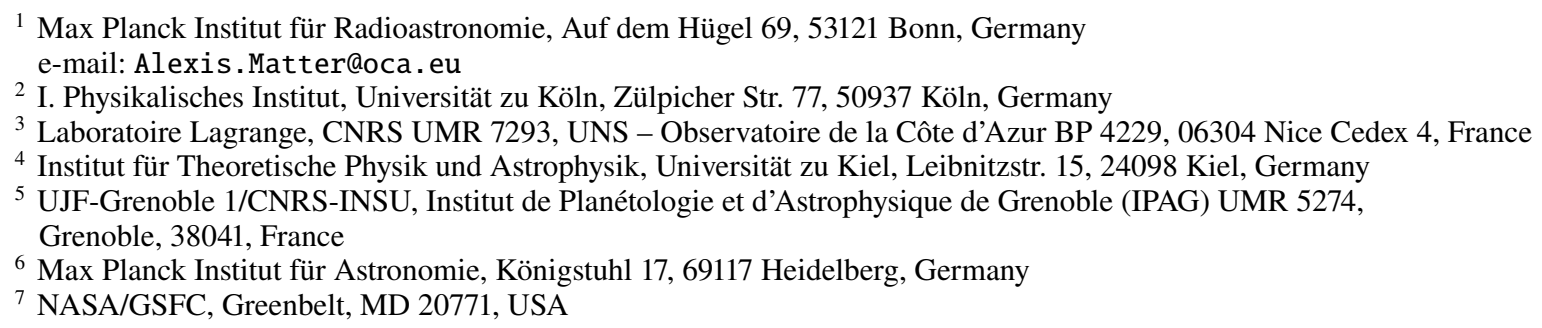

A\&A, 561, A26 (2014), https://doi .org/10.1051/0004-6361/201322042

Key words. instrumentation: high angular resolution - instrumentation: interferometers - techniques: interferometric stars: pre-main sequence - protoplanetary disks - errata, addenda

The original article contains three typos, which are corrected below.

Section 4.2: a factor $\frac{1}{d^{2}}$ is missing in the expression (2) of the visibility. The correct expression for the visibility to consider is:

$$
\begin{aligned}
V_{\lambda, \text { disk }}\left(B_{\mathrm{p}}(i, \theta)\right)= & \frac{1}{F_{\lambda}(0)} \frac{1}{d^{2}} \int_{r_{\text {in }}}^{r_{\text {out }}} B_{\lambda}\left(T_{r}\right) \epsilon_{\tau} J_{0} \\
& {\left[\frac{2 \pi}{\lambda} B_{\mathrm{p}}(i, \theta) \frac{r}{d}\right] 2 \pi r \mathrm{~d} r . }
\end{aligned}
$$

Section 4.2.1: a modulus symbol is missing at the numerator of the expression (6) of the visibility. The correct expression for the visibility to consider is:

$V_{\mathrm{tot}, 1}=\frac{\left|F_{*}(\lambda)+F_{\lambda, \text { disk }}(i) V_{\lambda, \text { disk }}\left(B_{\mathrm{p}}(i, \theta)\right)\right|}{F_{*}(\lambda)+F_{\lambda, \text { disk }}(i)}$.
Section 4.2.2: a modulus symbol is missing at the numerator of the expression (11) of the visibility. The correct expression for the visibility to consider is:

$$
\begin{aligned}
& V_{\mathrm{tot}, 2}= \\
& \frac{\left|F_{*}(\lambda)+F_{\lambda, \text { disk }_{\mathrm{h}}}(i) V_{\lambda, \text { disk }_{\mathrm{h}}}\left(B_{\mathrm{p}}(i, \theta)\right)+F_{\lambda, \text { disk }}(i) V_{\lambda, \text { disk }}\left(B_{\mathrm{p}}(i, \theta)\right)\right|}{F_{*}(\lambda)+F_{\lambda, \text { disk }_{\mathrm{h}}}(i)+F_{\lambda, \text { disk }}(i)} .
\end{aligned}
$$

All the visibility calculations presented in the original article were based on the correct expressions above. 